\title{
Evidence That Long-Term Potentiation Occurs within Individual Hippocampal Synapses during Learning
}

\author{
Vadim Fedulov, ${ }^{1}$ Christopher S. Rex, ${ }^{2}$ Danielle A. Simmons, ${ }^{3}$ Linda Palmer, ${ }^{4}$ Christine M. Gall, ${ }^{1,2}$ and Gary Lynch ${ }^{3}$ \\ Departments of ${ }^{1}$ Anatomy and Neurobiology, ${ }^{2}$ Neurobiology and Behavior, and ${ }^{3}$ Psychiatry and Human Behavior, University of California, Irvine, \\ California 92617-4291, and ${ }^{4}$ Department of Philosophy, Carnegie Mellon University, Pittsburgh, Pennsylvania 15213
}

\begin{abstract}
Stabilization of long-term potentiation (LTP) depends on multiple signaling cascades linked to actin polymerization. We used one of these, involving phosphorylation of the regulatory protein cofilin, as a marker to test whether LTP-related changes occur in hippocampal synapses during unsupervised learning. Well handled rats were allowed to explore a compartmentalized environment for $30 \mathrm{~min}$ after an injection of vehicle or the NMDA receptor antagonist ( \pm )-3-(2-carboxypiperazin-4-yl)propyl-1-phosphonic acid (CPP). Another group of rats consisted of vehicle-injected, home-cage controls. Vehicle-treated rats that explored the environment had $30 \%$ more spines with dense phosphorylated (p) cofilin immunoreactivity in hippocampal field CA1 than did rats in the home-cage group. The increase in pCofilin-positive spines and behavioral evidence for memory of the explored environment were both eliminated by CPP. Coimmunostaining for pCofilin and the postsynaptic density protein 95 (PSD-95) showed that synapses on pCofilin-positive spines were substantially larger than those on neighboring (pCofilin-negative) spines. These results establish that uncommon cellular events associated with LTP, including changes in synapse size, occur in individual spines during learning, and provide a technique for mapping potential engrams.
\end{abstract}

Key words: cofilin; actin; PSD-95; phosphorylation; unsupervised learning; immunoreactivity

\section{Introduction}

Determining how and where the brain encodes memories are long-standing and central goals in behavioral neuroscience. Nineteenth century investigators deduced that memory formation involves building associations between "nervous elements" in the neocortex, producing novel combinations of cells (Ribot, 1882) and ultimately a trace, or "engram," spanning multiple brain regions (Semon, 1921; described by Schacter, 1982). Tests of whether learning establishes functional networks (Olds et al., 1972; Gamlin et al., 1984) eventually led to the discovery that eye-blink conditioning uses defined anatomical pathways to carry information about conditioned and unconditioned stimuli, with convergence and encoding occurring in a cerebellar nucleus (Krupa et al., 1993). Other work provided physiological evidence for synaptic changes in amygdala during fear conditioning (Blair et al., 2005).

An alternative approach to localizing the engram involves first identifying variants of synaptic plasticity that are plausible candidates for the memory substrate, and then testing for their presence after learning. Increasing interest in long-term potentiation (LTP) has motivated much research related to this "bottom-up"

Received May 2, 2007; revised June 14, 2007 ; accepted June 14, 2007.

This work was supported by National Institute of Neurological Disorders and Stroke Grants NS051823 and NS045260. C.S.R. was supported by National Institute on Aging Training Grant 5T32-AG00358. We thank Nha Pham and Lulu Chen for assistance with immunocytochemistry, Natasha Narayan for behavior testing, and Yong Park and Katie Stribling for confocal microscopy. The Bio-Rad Radiance 2000 confocal microscope system was provided by the University of California, Irvine, Institute for Brain Aging and Dementia.

Correspondence should be addressed to Gary Lynch, 101 Theory Drive, \#250, University of California, Irvine, CA 92612-1695. E-mail: glynch@uci.edu.

DOI:10.1523/JNEUROSCI.2003-07.2007

Copyright $\odot 2007$ Society for Neuroscience $\quad$ 0270-6474/07/278031-09\$15.00/0 strategy. Numerous parallels between LTP and memory have been identified, and manipulations have been found that selectively disrupt both potentiation and the encoding of new information (Bliss and Collingridge, 1993). Although results of these types led to the widespread assumption that LTP is intimately involved in memory storage, they did not provide means for localizing it after learning, an essential step for engram research.

Initial efforts to test for LTP in defined synaptic populations during learning used patterned stimulation of cortical afferents as conditioned stimuli, and asked whether acquisition of electrical "cues" is accompanied by an increase in monosynaptic field EPSPs evoked by single stimulation pulses (Roman et al., 1987). Positive results were obtained and confirmed in other experiments of this type (Doyere and Laroche, 1992). A more recent paradigm used multiple electrodes to show that the relative magnitudes of field EPSPs recorded at several sites within hippocampus, and evoked by a single stimulation site, are modified by learning (Whitlock et al., 2006). These results provide good evidence that acquisition of cues changes synaptic weights. We extended this line of research in the present study to directly visualize LTP-related changes to the architecture of individual synapses during learning.

Previous work with hippocampal slices has provided several potential markers for the occurrence of LTP in vivo. Briefly, the naturalistic theta burst pattern of afferent stimulation causes actin polymerization in a subpopulation of spine heads in fields of potentiated synapses (Lin et al., 2005). This effect and LTP have the same threshold, stabilize over the same time period, and are disrupted by the same manipulations (Kramar et al., 2006). Like LTP, theta stimulation-induced actin polymerization depends on integrins and brain-derived neurotrophic factor (Kramar et al., 
2006; Rex et al., 2007) and is accompanied by phosphorylation of their downstream effectors in the p21-activated kinase-cofilin cascade (Chen et al., 2007). Dendritic spines in which this machinery has been activated have postsynaptic densities (PSDs) that are much larger than their neighbors (Chen et al., 2007). Here we use double-immunolabeling for PSDs and pCofilin to show that learning activates, within single synapses, the same consolidation processes found with LTP.

\section{Materials and Methods}

Animals. All animal procedures were conducted in accordance with the National Institutes of Health Guide for the Care and Use of Laboratory Animals and with protocols approved by the Institutional Animal Care and Use Committee of the University of California, Irvine. Adult (200$300 \mathrm{~g}$ ) male Sprague Dawley rats (Charles River, Gilroy, CA) were group housed under a reverse light/dark cycle (2 P.M. to 2 A.M.) and given food and water ad libitum.

Behavioral apparatus and unsupervised learning paradigm. The behavioral test apparatus (see Fig. 1) consisted of a large open field attached to a smaller, enclosed dark compartment accessible to the animal by an open entrance (total apparatus size in inches, 18 wide $\times 30$ long $\times 15$ high). Infrared light, which rats do not detect (Neitz and Jacobs, 1986), illuminated the otherwise dark compartment through a visually opaque but infrared transparent acrylic cover. Two exterior walls (both adjacent to the corner) (see Fig. 1 A, i) of the weakly lit open field were transparent, permitting the animal to view cues in the test room outside the behavior apparatus. Two objects of approximately equal size were placed in the open field (see Fig. $1 \mathrm{~A}$, i small gray circles in fields $\mathrm{d}$ and e).

Behavior was recorded during the entire testing period by an overhead camera (Axis 207W; Axis Communications, Lund, Sweden). Movements were tracked and analyzed using TopScan 1.0 Software (CleverSys, Reston, VA) at nine frames per second to measure the following variables over time ( 5 min bins): (1) time spent in the dark compartment and open field; (2) total number of movements; (3) duration of movements; (4) number of approaches (within $100 \mathrm{~mm}$ ) to each object; (5) time spent adjacent to each object. A movement was defined as instantaneous speed over threshold $(60 \mathrm{~mm} / \mathrm{s})$ maintained for at least $0.67 \mathrm{~s}$. Two subzones in the dark compartment (see Fig. 1A, a, b, near the back and near the entrance to the open field, respectively) and seven subzones in the open field (Fig. 1, h, near the entry; d, e, area surrounding the objects; g, i, and $j$, in corners of open field; $f$, near the far wall overlooking the testing room; and c, in the remaining open field) were defined, and counts, durations, and numbers of visits to each zone were quantified. Patterns of visits to the subzones lasting longer than $1 \mathrm{~s}$ were analyzed using custom scripts written in the open-source $\mathrm{R}$ statistical programming language ( $\mathrm{R}$ Foundation for Statistical Computing, Vienna, Austria; www.rproject.org) and other measures were analyzed using Excel (Microsoft, Redmond, WA).

Rats were handled and administered vehicle injections daily for $5 \mathrm{~d}$ before the onset of behavioral testing. They were subjected to the unsupervised learning task for 2 consecutive days. Experimental rats received intraperitoneal injection of $0.9 \%$ saline (vehicle, $n=8)$ and $( \pm)-3-(2-$ carboxypiperazin-4-yl)propyl-1-phosphonic acid (CPP) rats received intraperitoneal injection of $10 \mathrm{mg} / \mathrm{kg} \mathrm{CPP}(n=6)$ in saline $2 \mathrm{~h}$ before testing on day 1 only. On both test days, rats were transported to the test room in an opaque box. Both experimental and CPP rats were introduced into the environment at the same location directly between the open field and the "home box" and allowed to freely explore the environment for $30 \mathrm{~min}$. The boxes/objects were cleaned with mild detergent/water solution between rats and with SCOE 10X odor/urine eliminator (BioFog, Alpharetta, GA) after each test day.

For a subset of vehicle-injected, experimental rats $(n=5)$, object substitution was performed on a third consecutive behavioral test day by replacing one of the objects in the open field with a novel object of similar size; paired control rats for the object recognition test $(n=5)$ received the same object on day 3 .

For immunocytochemical analyses, a separate set of rats were divided into experimental $(n=14)$, CPP-treated $(n=8)$, and home-cage control
(HC; $n=10)$ groups, subjected to the behavior test, and then killed. As described above, experimental and CPP rats performed the unsupervised learning task after vehicle or CPP injections (i.p.), respectively. The HC rats received a vehicle injection and were transported to the test room, but were not placed into the behavioral apparatus; they were then returned to their home cage and killed 30 min later. Immunocytochemistry for pCofilin and PSD-95 was performed on four batches of tissue. Each batch of simultaneously processed tissue included sections from experimental rats ( $n=2-4 / \mathrm{batch})$ and sections from the HC or CPP (or both) groups ( $n=2-4 /$ batch/group). Because all batches contained tissue from the experimental group, quantification of immunoreactive puncta within a batch was normalized to the experimental group mean for that batch.

Immunocytochemistry. Rats were anesthetized with halothane inhalant anesthesia and perfused with $4 \%$ paraformaldehyde in $0.1 \mathrm{~m}$ sodium phosphate buffer (PB), pH 7.2. Brains were postfixed for $2 \mathrm{~h}$, cryoprotected in $20 \%$ sucrose/PB, and then sectioned $(25 \mu \mathrm{m})$ on a freezing microtome. Free-floating sections were processed for doubleimmunolabeling using rabbit anti-cofilin (1:250; Cytoskeleton, Denver, CO) or anti-pCofilin [pS3] IgG (1:100; catalog \#12866; Abcam, Cambridge, MA), and mouse anti-PSD-95 (1:1000; \#MA1-045, Affinity Bioreagents, Golden, CO). Secondary antisera included Alexa Fluor 555 anti-rabbit IgG and Alexa Fluor 488 anti-mouse IgG (both 1:200; Invitrogen, Carlsbad, CA) in PB. Control tissue was processed by the same procedures but with exclusion of one or the other primary antisera from the initial incubation: under these conditions the only labeling observed was associated with the primary antibody.

Confocal microscopy and measurement of labeled profiles. Laserscanning confocal microscopy was performed using the Bio-Rad Laboratories (Hercules, CA) Radiance 2000 Laser Scanning System using a $60 \times[1.4$ numerical aperture (NA)] Plan Apo objective. Optical sections $(1 \mu \mathrm{m})$ were scanned at $4 \times$ zoom in a $512 \times 512$ pixel format. Image montages covering a $205 \times 205 \mu \mathrm{m}$ area within the midproximal CA1 stratum radiatum were collected from eight sections (at regular intervals) from the midrostral to midcaudal portion of the hippocampus; in all, $\sim 300,000 \mu \mathrm{m}^{3}$ (area $\times 1 \mu \mathrm{m}$ optical section thickness) was examined for each rat. The entire image field was processed for signal quantification. Images were converted to grayscale and intensity levels were scaled to values (Photoshop CS, version 8.0; Adobe, San Jose, CA) appropriate for detecting low-intensity labeling. The "true" threshold for detecting immunostained puncta is somewhat lower than the levels used in experiments because it falls in the range at which visual noise begins to flood the image. Thresholds in practice were set at values just above that level at which diffuse, background fluorescence appeared. This necessarily excluded faintly labeled elements, which is why counts for spines and synapses in the immunocytochemical studies are inevitably smaller than those obtained with electron microscopy.

Tissue from all rats within a batch was run in parallel using the same intensity threshold. Analysis was conducted blind to sample identity on batches that had been processed together. Object analysis software, which is a mix of Perl and C (Lin et al., 2005; Chen et al., 2007; Rex et al., 2007), processed edge detection by imposing a binarized edge map across the image to count and measure the area of labeled structures. Intensity thresholds ( 8 bits/pixel) were applied within a range that reliably identified objects. Pixel values for each image were normalized to reduce the impact of background intensity differences across the image, binarized using the specified intensity threshold, and finally cleaned by "erosion" and "dilation" filtering (Jain, 1984). These procedures use preset criteria to remove unconnected, presumably spurious pixels and to fill small gaps and smooth contours, respectively. Finally, ellipticity of each object was determined to identify only disk-like structures. Identified objects $<0.04$ $\mu \mathrm{m}^{2}$ and $>1.2 \mu \mathrm{m}^{2}$ were excluded from analysis. Counts from multiple sections were averaged to produce a representative value for each rat. Colocalization of immunostaining was determined automatically (Matlab; Mathworks, Natick, MA) using each identified object's center coordinates and radius to determine overlap.

Widefield microscopy and three-dimensional reconstruction. Widefield photomicrographs of immunostained tissue were acquired with a Leica (Bannockburn, IL) DM6000 B microscope, using a 63× Plan Apo (1.4 
NA) objective. Z-series ( $0.2 \mu \mathrm{m}$ steps) images were deconvolved by iterative restoration using Volocity 4.0 Restoration software (Improvision, Lexington, MA). Three-dimensional reconstructions of the target field were generated and qualitatively analyzed for overlap between pCofilinand PSD-95-immunoreactive elements.

Statistical analyses. Statistics were computed with Microsoft Excel or with Prism version 5 software (GraphPad, San Diego, CA). Analyses of behavioral experiments were performed by conventional ANOVA, repeated-measures ANOVA (RM-ANOVA), or Student's $t$ test (twotailed unless otherwise noted). Statistical significance of puncta counts was determined by Student's $t$ test (two-tailed unless otherwise noted). Group puncta size distributions were compared using the KolmogorovSmirnov $Z$ test or a conventional test for skewedness. All measures are reported as group means \pm SD in text and as means \pm SEM in figures.

\section{Results}

\section{Behavior in an unsupervised learning task}

The design of this study involved a test of the predictions that a 30 min period of unsupervised learning in an environment with objects and spatial cues would produce evidence of LTP in hippocampal CA1 stratum radiatum, and that such effects would be impaired by blocking NMDA receptors. Accordingly, initial behavioral experiments assessed the degree to which the NMDA receptor antagonist CPP disrupts the encoding of long-term memory in an unsupervised learning task. CPP has been shown to block LTP induction in vivo (Davis et al., 1997) and to impair behavioral measures of memory (Ward et al., 1990; Mele et al., 1996).

Well-handled rats were placed in a compartmentalized field (Fig. 1, ) and movements were monitored for $30 \mathrm{~min}$. The total number of movements by rats given vehicle injections (experimentals) decreased steadily within a 30 min test session on day 1 (first 5 vs last 5 min bins; $p=0.0004 ; t$ test) (Fig. $2 A$ ). Similar results were obtained on day $2(p=0.004)$. There was also clear evidence that the experience of day 1 extended to day 2: the number of movements during the first $20 \mathrm{~min}$ of exploration dropped by $25 \pm 26 \%$ ( $p=0.02$, paired $t$ test $)$ from the first to the second day of testing (Fig. $2 \mathrm{~B}$ ). There were also significant reductions in the duration of visits to the objects during the first $20 \mathrm{~min}$ $(-37 \pm 20 \% ; p=0.0004$ for day 1 vs day 2 , paired $t$ test) (Fig. $2 C)$ and the total time spent in proximity to the objects $(p<0.001)$ (Fig. 2D).

Rats injected with CPP performed similar numbers of movements as those rats given vehicle during the first day of testing (Fig. 2A) and exhibited comparable decreases in activity over time ( $p=0.0001$ for the first vs last 5 min bins, paired $t$ test). Moreover, the mean duration of individual movements during this period did not differ between groups (experimental, $2.0 \pm$ $0.2 \mathrm{~s}$; CPP, $1.8 \pm 0.2 \mathrm{~s} ; p=0.13$, unpaired $t$ test). In contrast, the carry-over effects from day 1 to day 2 found in vehicle-treated rats did not occur in CPP-treated rats. The numbers of movements per 5 min during the first $20 \mathrm{~min}$ of exploration for CPP rats on day $2(32 \pm 1)$ was comparable with that on day $1(27 \pm 6)$ (Fig. $2 B)$. Similarly, the mean duration of visits to the open field objects was equivalent between testing days $(2.0 \pm 1.3$ on day 1 and $2.0 \pm 1.0 \mathrm{~s}$ on day 2 ) (Fig. $2 C$ ), as was the total time spent near the objects (188 \pm 56 and $172 \pm 56 \mathrm{~s}$ ) (Fig. $2 \mathrm{D})$.

The above results, which accord with previous findings (Ward et al., 1990; Mele et al., 1995, 1996), constitute clear evidence that CPP blocks the formation of long-term memory during testing on day 1 , and does so without substantially changing overall levels of activity or their decrease with time. However, examination of day 1 activity patterns indicated that CPP did disrupt normal behavior (Fig. 3). The distribution of entries into subfields, be-
A.

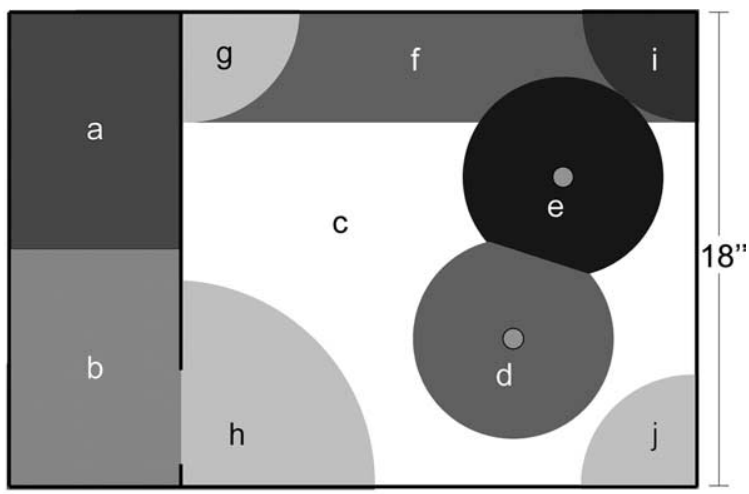

$6.5^{\prime \prime}$

$30 "$

B.

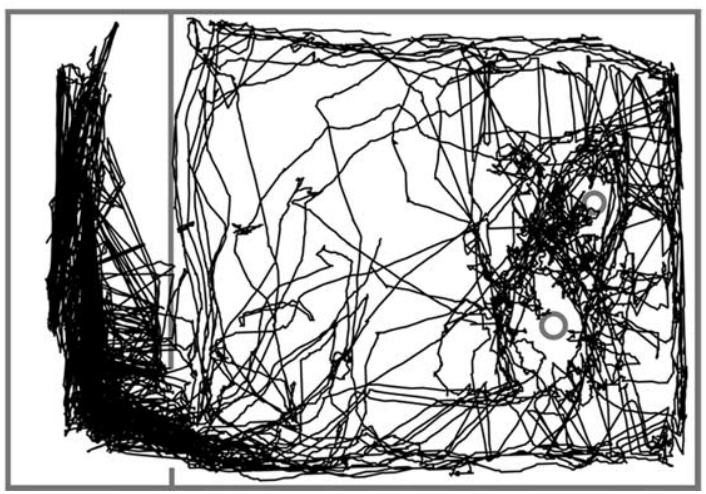

Figure 1. Behavioral apparatus to assess unsupervised learning. $A$, Schematic of behavioral apparatus floor plan shows delineation of subzones (a-j) used for analysis of motion tracking data in the unsupervised learning task. Two objects placed in the open field compartment are identified by small gray circles within fields labeled $d$ and e. Gray regions identify areas of the multicompartment environment: dark compartment $(a, b)$, dark entry (h), open field (c), corners $(g, i, j)$, window $(f)$, and area surrounding objects (d, e). $\boldsymbol{B}$, Representative trace of rat locomotion during the 30 min test on day 1 in the unsupervised learning environment generated from video analysis by motion-tracking software. Boundaries of the behavioral apparatus and locations of objects are shown as gray lines.

tween visits of $\geq 1 \mathrm{~s}$, expressed as a percentage of all such actions, was comparable across rats in the experimental group (Fig. $3 A$ ). CPP altered this pattern from the start of testing (Fig. $3 A$ ): the difference between groups in the distribution of entries into the nine subfields on day 1 was highly significant $(p<0.0001$ for interaction terms in a two-way RM-ANOVA), with the main effect attributable to CPP-treated rats spending more time in the dark compartment.

Zone-to-zone analyses indicated that experimental rats gradually shifted their exits from sites in the "open space" (all areas outside the object fields, entrance zone, and dark box) away from the dark compartment entrance to other positions in the open space (Fig. 3B), an effect that persisted to the second day of testing. Analysis of this measure for CPP rats was not possible because they spent too little time in the open space (Fig. 3A). Finally, work with a separate group of experimental rats confirmed that replacing one of the open field objects between days of testing increased object exploration (Fig. 3C).

\section{Cofilin and pCofilin are localized to excitatory synapses}

Cofilin removes actin monomers from the sharp end of growing actin filaments (Sarmiere and Bamburg, 2004). This activity is 
A. within day 1

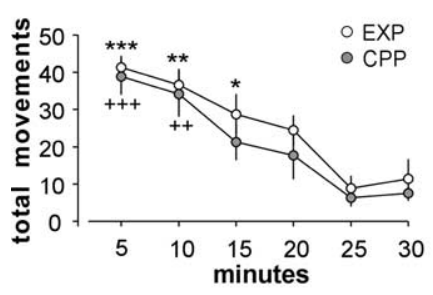

C.

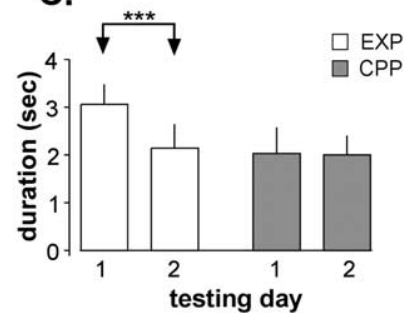

B. between days 1 and 2

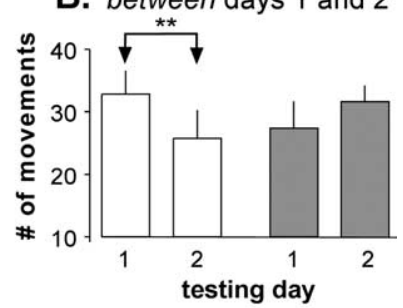

D.

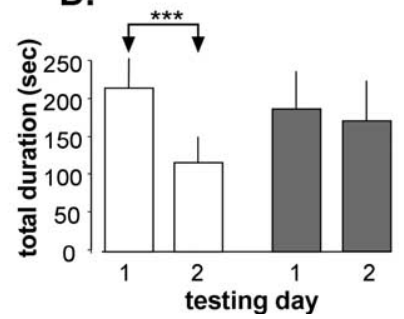

Figure 2. Behavioral analysis of experimental (vehicle-treated) and (PP-treated rats in a multicompartment environment. $\boldsymbol{A}$, Numbers of movements (30 min session, 5 min bins) on day 1 did not differ between (PP and experimental (EXP) rats; these groups exhibited comparable decreases in activity over the test session $\left({ }^{* * *} p=0.0004,{ }^{* *} p=0.002,{ }^{*} p=0.05\right.$ vs 30 min of the EXP group; ${ }^{++} p=0.0001,{ }^{++} p=0.008$ vs 30 min of the (PP group). $\boldsymbol{B}$ Numbers of movements for the first 20 min of testing on day 1 versus the same period on day 2 . The mean number of events decreased on day 2 in EXP but not CPP-treated rats ( ${ }^{* *} p=0.02$ ). C, Mean duration of visits to the object area during the first 20 min of testing on days 1 and 2 . This value, which was not correlated with numbers of movements $(r=0.26)$, decreased between the $2 \mathrm{~d}$ for EXP but not (PP rats $\left({ }^{* * *} p<0.001\right)$. D, Total time visiting the objects during days 1 and 2 . This measure, which combines number and mean duration of visits, decreased for EXP but not for CPP rats $\left({ }^{* *} p=0.001\right)$.

suppressed when cofilin is phosphorylated, thereby creating conditions that are favorable for cytoskeleton assembly (Juliano et al., 2004; Sarmiere and Bamburg, 2004; Carlisle and Kennedy, 2005). Cofilin is mostly restricted to dendritic spines (Racz and Weinberg, 2006) and plays a major role in regulating spine anatomy in developing neurons (Hayashi et al., 2004; Zhou et al., 2004; Boda et al., 2006). In agreement with this localization, discrete puncta containing immunoreactivity (ir) for total cofilin (i.e., both phosphorylated and unphosphorylated) were present in large numbers $\left(14 \pm 4\right.$ spines/100 $\mu^{3}$ ) throughout all hippocampal dendritic lamina (Fig. 4A). The size, shape, and distribution of cofilin-immunoreactive puncta strongly suggested that they are dendritic spine heads (Fig. $4 A$, inset). Spines with dense pCofilin immunoreactivity were much less numerous $(1.4 \pm 0.6$ spines $/ 100 \mu \mathrm{m}^{3}$ ) (Fig. $4 B$; supplemental Fig. 1, available at www. jneurosci.org as supplemental material), as shown previously in acute hippocampal slices (Chen et al., 2007). Thus, $\sim 10 \%$ of the densely cofilin-ir spines contain substantial pCofilin-ir when the animal is within its normal laboratory housing conditions.

Localization of pCofilin to spines was confirmed by its juxtaposition with structures that were $\sim 0.5 \mu \mathrm{m}$ in diameter and densely immunoreactive for the integral postsynaptic density protein PSD-95 (Hunt et al., 1996; Petersen et al., 2003) (Fig. $4 B-D)$. Restorative deconvolution analyses showed that the PSD-95 and pCofilin-positive (pCof + ) structures only partially overlapped and had different shapes (Fig. $4 E$ ). This is entirely consistent with immunolabeling of the subsynaptic scaffold (PSD-95) and a protein located slightly further back in the spine head (pCofilin).

Together, the above results indicate that cofilin is ubiquitously present in spines throughout stratum radiatum, but is extensively phosphorylated in only a small fraction of these structures.

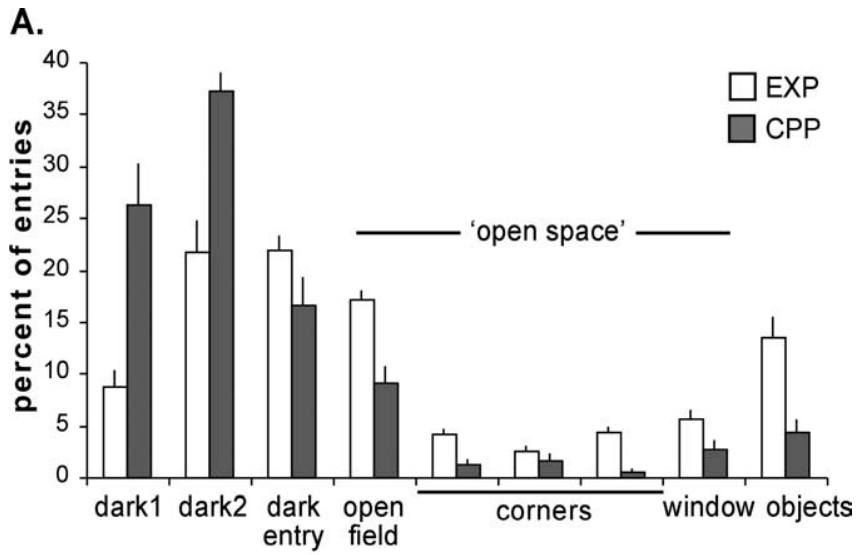

B.

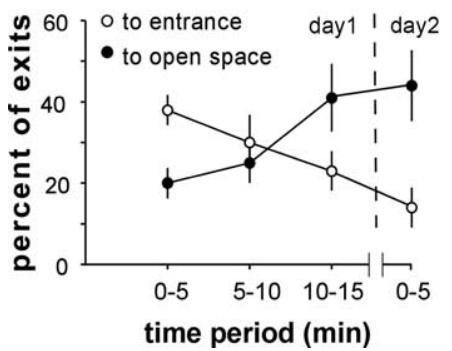

C.

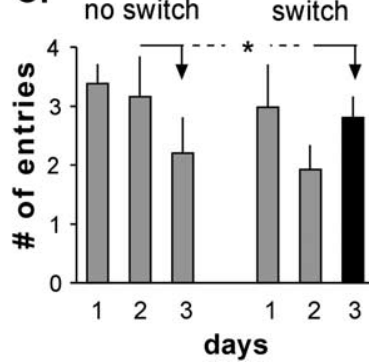

Figure 3. CPP disrupts normal patterning of activity in a multicompartment environment. $\boldsymbol{A}$, The distribution of movements (between stays of $\geq 1 \mathrm{~s}$ ) into different locations of the environment, expressed as a percentage of all such actions during initial exploration. The difference between patterns for experimental (EXP; vehicle-injected) and CPP groups was highly significant ( $p<0.0001$ for interaction terms in a two-way RM-ANOVA), with the main effect arising from time spent in dark compartments 1 and 2. $\boldsymbol{B}$, The distribution of movements between specific locations changed across days in EXP rats: Shown are exits from the "open space" (corners, region next to a window, open field) region of the environment. EXP rats initially returned to the entrance area of the dark box then began distributing movements to other sites in the open space during day 1 (left side of graph); the altered behavioral pattern persisted on day 2 (right side). C, Replacing one of the open field objects increased object exploration in EXP rats. Rats were tested as above, except that a third day of testing was added, on which one-half of the rats $(N=5)$ received the same objects (no switch) and the other half $(N=5)$ had a novel object substituted for one of the old ones (switch; black bar). The replacement group increased object exploration from days $2-3$ whereas the same-objects group did not $\left({ }^{*} p=0.016\right.$, MannWhitney $U$ test, with direction predicted, for comparison of day 2 to day 3 deltas for the two groups).

\section{Unsupervised learning leads to increases in the number of pCofilin-positive spines}

Previous experiments with hippocampal slices showed that theta burst stimulation produces a marked, region-specific increase in the number of spines having both dense concentrations of $\mathrm{pCo}-$ filin and larger than normal synapses (Chen et al., 2007). We tested whether unsupervised learning produces similar effects.

Well-handled rats were randomly assigned to one of three groups: (1) vehicle-treated exploration rats (experimental group), (2) CPP-treated exploration rats, and (3) HC controls (rats injected with vehicle but not placed in the open field). Exploration (and paired HC) rats were killed $30 \mathrm{~min}$ after placement in the test apparatus and tissue sections through hippocampus were processed for PSD-95 and pCofilin immunohistochemistry in sets. The mean area of CA1 stratum radiatum sampled per rat was 300,000 $\mu \mathrm{m}^{2}$, which, given that $1 \mu \mathrm{m} \mathrm{z}$-stacks were used in all cases, converts to an equivalent $\mu \mathrm{m}^{3}$ value. Counts (per $100 \mu \mathrm{m}^{3}$ ) for a given rat were normalized to the mean of the experimental group within the same set.

Rats in the experimental group had substantially more densely 

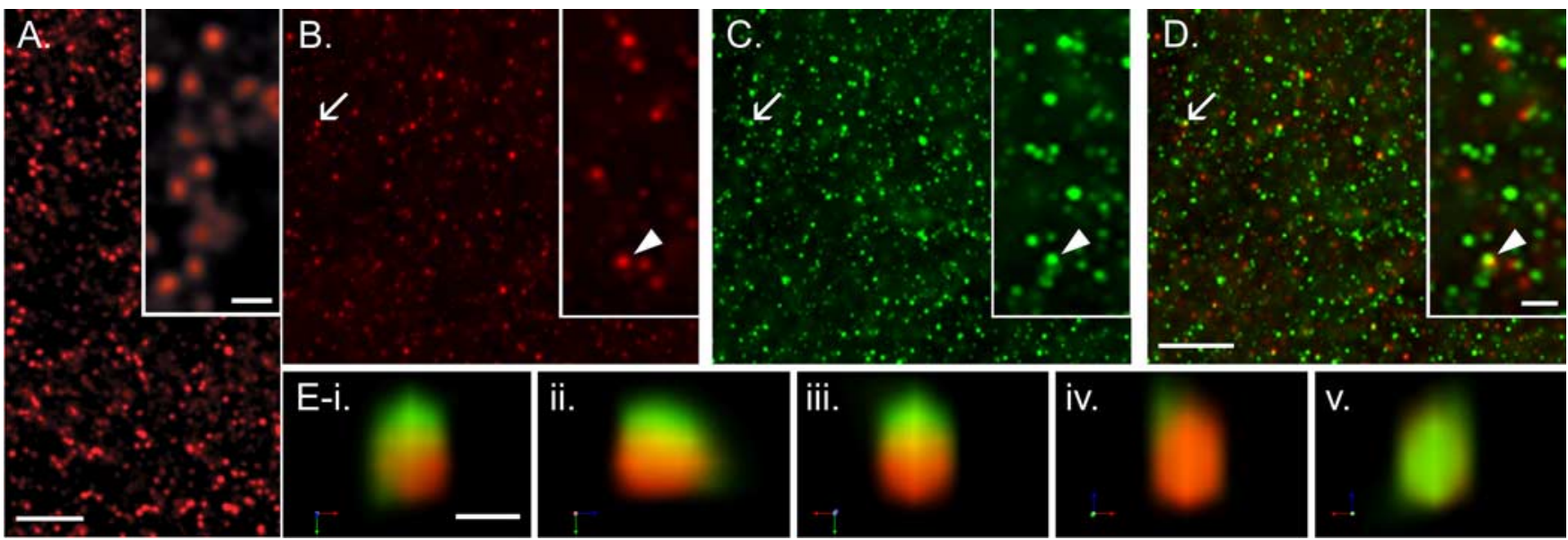

Figure 4. Cofilin, pCofilin, and PSD-95 immunostaining in CA1 stratum radiatum. $A$, Cofilin immunoreactivity was abundant in discrete puncta but was absent from cell bodies (data not shown) and dendrites: the size and shape of cofilin-ir puncta correspond to those for spine heads (inset). $\boldsymbol{B}$, Densely pCofilin-ir puncta were qualitatively similar to those labeled for total cofilin ( $\boldsymbol{A}$ ) but were present in smaller numbers. C, PSD-95-ir profiles were numerous. D, Merged images confirm that pCofilin-ir profiles are associated with PSD-95-ir puncta. The arrows and arrowheads point to the same sites in $\boldsymbol{B}-\boldsymbol{D}$ to show spatial relationship and overlap. $\boldsymbol{E}$, Three-dimensional reconstruction from restorative deconvolution microscopy shows the association between pCofilin-ir (red) and PSD-95-ir puncta (green; overlap appears yellow). Modeling of labeled puncta in reference to view irotation $90^{\circ} y$-axis (ii), $180^{\circ} y$-axis (iii), $90^{\circ} x$-axis bottom (iv), and $90^{\circ} x$-axis top ( $v$ ) reveals strong anatomical coupling between pCofilin and PSD-95. Scale bars: $\boldsymbol{A}, 5 \mu \mathrm{m}$; inset, $1 \mu \mathrm{m}$; (in $\boldsymbol{D}) \boldsymbol{B}-\boldsymbol{D}, 10 \mu \mathrm{m}$; insets, $2 \mu \mathrm{m} ; \boldsymbol{E}, 0.5 \mu \mathrm{m}$.

\section{A. $\mathrm{pCof}^{+}$spines}

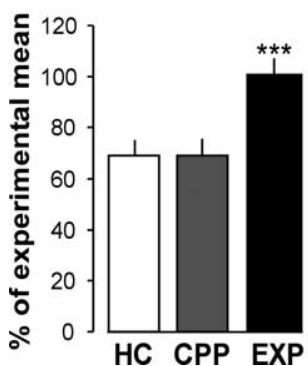

B. PSDs
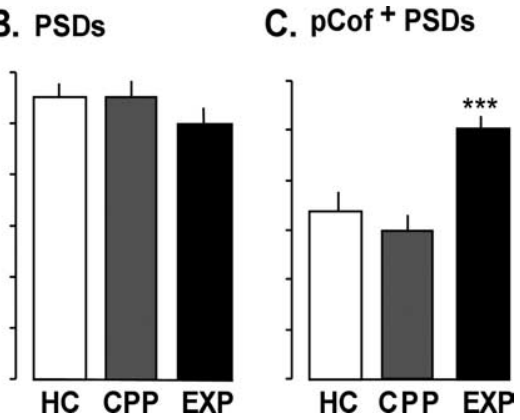

Figure 5. The number of spines with dense pCofilin-ir is dependent on behavior. Spines with dense concentrations of $\mathrm{pCofilin}(\mathrm{pCof}+$ ) were counted and values for a rat in a given cohort were normalized to the mean score for experimental (EXP) rats in that cohort. $A$, Rats that explored the environment (EXP group; $n=14$ ) had $\sim 30 \%$ more $\mathrm{pCof}+$ spines than did home cage controls (HC; $n=10)$; this effect was absent in CPP rats (CPP; $n=8)$. Differences in $p C$ of + puncta between EXP versus (PP or HC groups were highly significant $\left({ }^{* * *} p<0.001\right)$. $B$, The increase in numbers of pCof + puncta in EXP compared with CPP and $\mathrm{HC}$ groups was not accompanied by an increase in total numbers of PSD-95+ puncta. $C$, The number of PSD-95+ puncta that were colocalized with pCofilin-ir was expressed as a percentage of the total PSD$95+$ puncta for each rat in the study (values were then normalized to the within-cohort EXP rat mean). The EXP group had a higher percentage of PSD-95 + puncta colocalized with $p$ Cofilin and than did either HC ( $p=0.003)$ or CPP groups ( $p=0.0004$; $t$ tests with direction predicted).

labeled pCofilin spines than did those in HC or CPP groups (Fig. $5 A$ ). Mean (normalized) numbers of pCof + spines for HC, CPP, and experimental groups were $69 \pm 23(n=10), 67 \pm 21(n=8)$, and $100 \pm 18(n=14)$, respectively; tests of the two experimental predictions were highly significant (experimental $>\mathrm{HC}, p=$ 0.0003; experimental $>$ CPP, $p<0.0003, t$ tests with direction predicted; group effect with ANOVA, $p=0.0003)$. Although these relative effects were robust, the $30 \%$ increase in pCof + spines found in the experimental rats involved only a small fraction of the synaptic population. HC rats had an average of $1.4 \pm$ 0.6 such spines per $100 \mu \mathrm{m}^{3}$, a value that is substantially less than that for the total number of synapses estimated to be present in a sample of this size from the apical dendrites of rat field CA1 $\left(\sim 300 / 100 \mu \mathrm{m}^{3}\right)$, as assessed with electron microscopy.

Analyses of the type just described indicated that learning did not increase the total number of synapses (Fig. 5B). The mean number of PSD-95+ puncta was similar in each group (HC, $111 \pm 20$; CPP, $110 \pm 17$; experimental, $100 \pm 14$ ). Finally, expressing the number of PSD-95+ puncta double-labeled for pCofilin as a percentage of the total number of PSD-95+ profiles ( $4.17 \pm 0.19 \%$ for HC group), and then normalizing as described above, produced the results summarized in Figure $5 C$. The mean percentage of PSDs associated with pCofilin was $\sim 35 \%$ greater in the experimental group than in the other two groups ( $p=0.003$ vs $\mathrm{HC}$ and $p=0.0004$ vs $\mathrm{CPP}$ ).

PCofilin-positive spines are associated with larger synapses Our previous analyses of acute hippocampal slices showing that LTP is accompanied by an increase in numbers of pCofilinenriched spines (Chen et al., 2007) also demonstrated that profiles immunoreactive for PSD-95, a protein that is specific to and uniformly distributed within PSDs of excitatory synapses (Hunt et al., 1996; Petersen et al., 2003), were larger on the pCof + spines than on their pCofilin-negative (pCof-) neighbors. The present study also obtained this result in hippocampus of rats subjected to the unsupervised learning task (Fig. 6). In the HC group, mean areas of PSD-95+ profiles were $0.35 \pm 0.13 \mu \mathrm{m}^{2}$ for pCof + profiles versus $0.21 \pm 0.06 \mu \mathrm{m}^{2}$ for pCof- elements $(p=$ 0.0005 , paired $t$ test). Similar differences were found in the CPP $\left(0.18 \pm 0.02\right.$ vs $\left.0.27 \pm 0.03 \mu \mathrm{m}^{2}, p=0.00001\right)$ and experimental $\left(0.21 \pm 0.04\right.$ vs $\left.0.39 \pm 0.15 \mu \mathrm{m}^{2}, p=0.00004\right)$ groups. It follows from these results that learning by increasing the number of pCofilin-enriched spines also increased the number of spines with large synapses.

Two additional analyses were conducted to test for factors that might distort measures of PSD size. First, to exclude the possibility that the labeling for PSD-95 and pCofilin somehow increased each other's apparent fluorescence, we tested whether the areas occupied by each marker covaried. There was no relationship between the sizes of the two colocalized elements $\left(r^{2}=0.0004\right)$ from a sample of $\sim 7000$ synapses, a result that agrees with previous findings in hippocampal slices (Chen et al., 2007). Second, we examined the relationship between area and mean fluorescence labeling intensity of PSD-95-ir puncta to exclude possible distortions in size measures arising as a function of differences in 


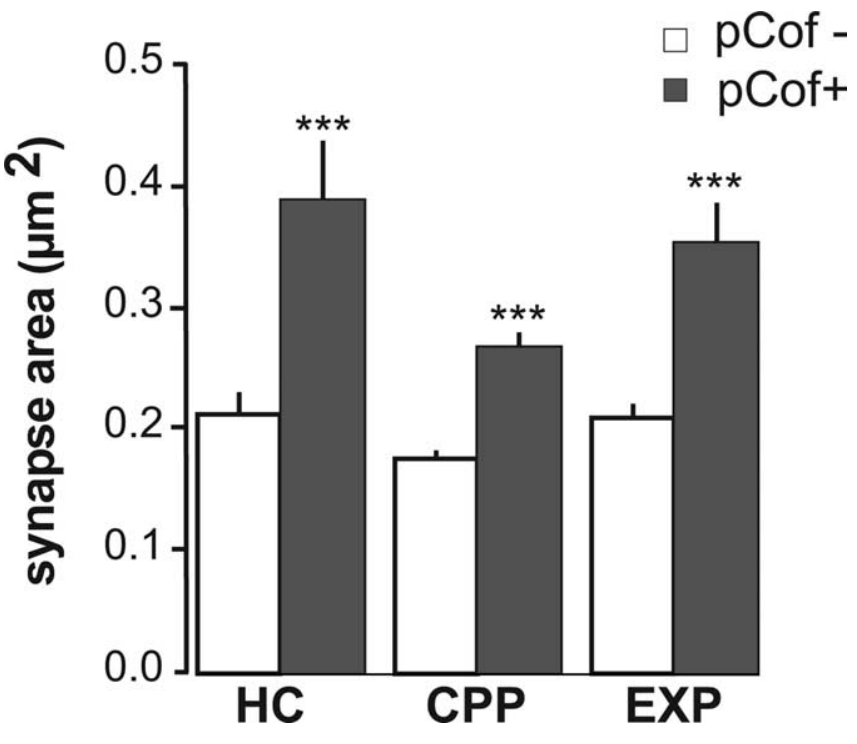

Figure 6. PSDs on pCof + spines were larger than those on $\mathrm{pCof}-$ neighbors. The PSD-95-ir puncta on pCof + spines were $78 \pm 36 \%$ (mean \pm SD) larger in the HC group, $52 \pm 12 \%$ larger in the CPP group, and $64 \pm 29 \%$ larger in the EXP group compared with pCof - puncta ${ }^{* * *} p<$ 0.0005 in all cases, two-tailed, paired $t$ tests).

labeling intensity. Figure 7 plots the relationship between PSD$95+$ object areas and log (base 2) mean object fluorescence intensity for $\sim 43,000$ identified synapses from both HC control and experimental rats; the two measures are unrelated $\left(r^{2}=\right.$ $0.13)$. These controls support the use of quantitative immunofluorescence for the determination of subcellular compartment sizes.

Data relevant to the reasons why synapses are larger on pCof + spines are summarized in Figure 8. Figure $8 A-C$ show the size distribution data for PSD-95+ puncta that were associated with pCof + and pCof - spines in the experimental rat closest to the group mean for synapse area. Note that the distribution of PSD areas for the infrequent pCof + synapses was shifted slightly to the right of that for the more numerous pCof- synapses (Fig. $8 \mathrm{~A}$ ), a point that is more clearly evident when data for particular size categories were expressed as a percentage of the total PSD population (Fig. $8 \mathrm{~B}$ ). Converting these data into a cumulative frequency curve (Fig. $8 C$ ) shows that the profile for pCof + synapses is not simply shifted relative to the curve for pCof- synapses, but instead exhibits a different distribution, as illustrated by subtraction of the two curves (inset). The deviations in cumulative frequency curves illustrated for the single experimental rat in Figure $8 A-C$ were reliably present for rats in all three groups (Fig. $8 D-F)$.

The $95 \%$ confidence intervals for the three sets of group data (Fig. $8 D-F$ ) indicate that the differences between cumulative frequency curves for PSD sizes were statistically robust. To provide a quantitative measure of this, we calculated, for each rat, the cumulative percentage of pCof- versus pCof + PSDs found in the $0.28 \mu \mathrm{m}^{2}$ size bin; these values were statistically different in the HC group ( $n=10 ; p<0.00001$, paired $t$ test). Similar values were obtained for CPP and experimental groups. As expected from this, distributions of raw pCof + versus pCof- scores, as illustrated in Figure $8 \mathrm{~A}$, were statistically different for nearly all rats in a given group. These observations indicate that the frequency distribution curves for the two classes of PSDs were reliably different. Finally, as implied by the above measurements, PSD distributions for pCof + spines were substantially less skewed than distributions for PSDs on pCof - spines (HC, $1.95 \pm$ 0.45 vs $1.19 \pm 0.65$ for $\mathrm{pCof}-\mathrm{vs} \mathrm{pCof}+, p=0.0001$; experimental, $1.89 \pm 0.44$ vs $1.18 \pm 0.61, p<0.00001$; paired $t$ tests $)$. These results indicate that the large PSDs colocalized with pCofilin are not simply expanded versions of isolated (pCof-) PSDs.

\section{Discussion}

The above findings demonstrate that learning a novel environment is accompanied by a sizable increase in the number of spines containing a marker for recently induced LTP. Theta burst stimulation, used to induce LTP, is known from previous work to cause cofilin phosphorylation in a small population of spines within the activated dendritic zone of hippocampal field CA1 (Chen et al., 2007). The effect was completely blocked by AMPA receptor antagonists, occurred within $2 \mathrm{~min}$ after stimulation, and dissipated within $30 \mathrm{~min}$ (Chen et al., 2007). Two lines of evidence suggest that the phosphorylation event is a step in the sequence leading to LTP consolidation. First, it is initiated by the neurotrophin brain derived neurotrophic factor (Rex et al., 2007), a modulatory factor released by theta burst stimulation that plays an important role in stabilizing LTP (Bramham and Messaoudi, 2005; Chen et al., 2007). Second, LTP consolidation is dependent on actin polymerization in spine heads (Fukazawa et al., 2003; Okamoto et al., 2004; Lin et al., 2005; Kramar et al., 2006), and actin filament assembly is known from many cell systems to be regulated by the phosphorylated state of cofilin (Sarmiere and Bamburg, 2004; Zhou et al., 2004). Importantly, in this study the learning-induced increase in the number of spines containing high concentrations of pCofilin was eliminated by a compound (CPP) that blocks NMDA receptors and thereby prevents LTP. To summarize, a process sensitive to the receptors and modulators that trigger LTP, and that is intimately related to the subsequent production of stable potentiation, occurs within hippocampal spines as rats acquire memories about a complex environment.

The links between pCofilin and LTP on the one hand, and LTP with memory on the other, suggest that the effects observed in the present study are directly related to the encoding of information. The finding that pCof + spines have unusually large synapses, a result first described for acute hippocampal slices (Chen et al., 2007) and replicated here in vivo, constitutes independent evidence for this conclusion. Specifically, results from multiple experiments indicate that AMPA receptors, which mediate fast EPSCs throughout the cortical telencephalon, increase in number as a function of synapse size (Nusser et al., 1998; Takumi et al., 1999; Racca et al., 2000; Ganeshina et al., 2004); thus, a conversion of a spine from a baseline state to the pCof + /large synapse condition would enhance the size of the synaptic current at that spine. Note also that only a small fraction of the total PSD population exhibited evidence of LTP-related effects, a result that satisfies the high capacity requirement for a memory mechanism in cortical systems (Granger et al., 1994).

The synaptic changes described here provide for rapid encoding of memory but additional mechanisms are likely required for long-term storage. Numerous studies indicate that protein synthesis is engaged by the triggering events for both LTP and memory and is required for their maintenance (for description, see Reymann and Frey, 2007). Learning, in this view, requires shortlatency cytoskeletal and synaptic changes in small populations of spines followed by transient, more global effects that reinforce these changes.

The larger-than-normal PSDs on pCof + spines did not appear to result from a simple expansion of synapses on pCof- 
A.
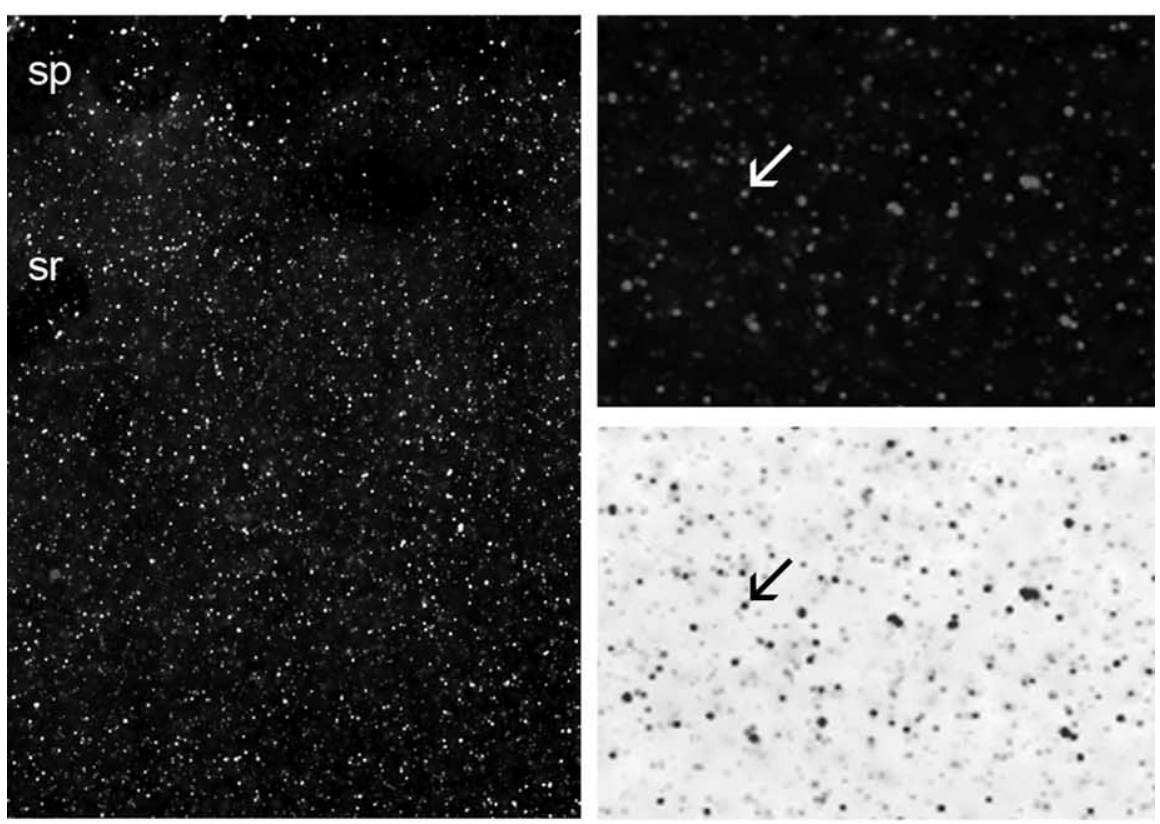

B.

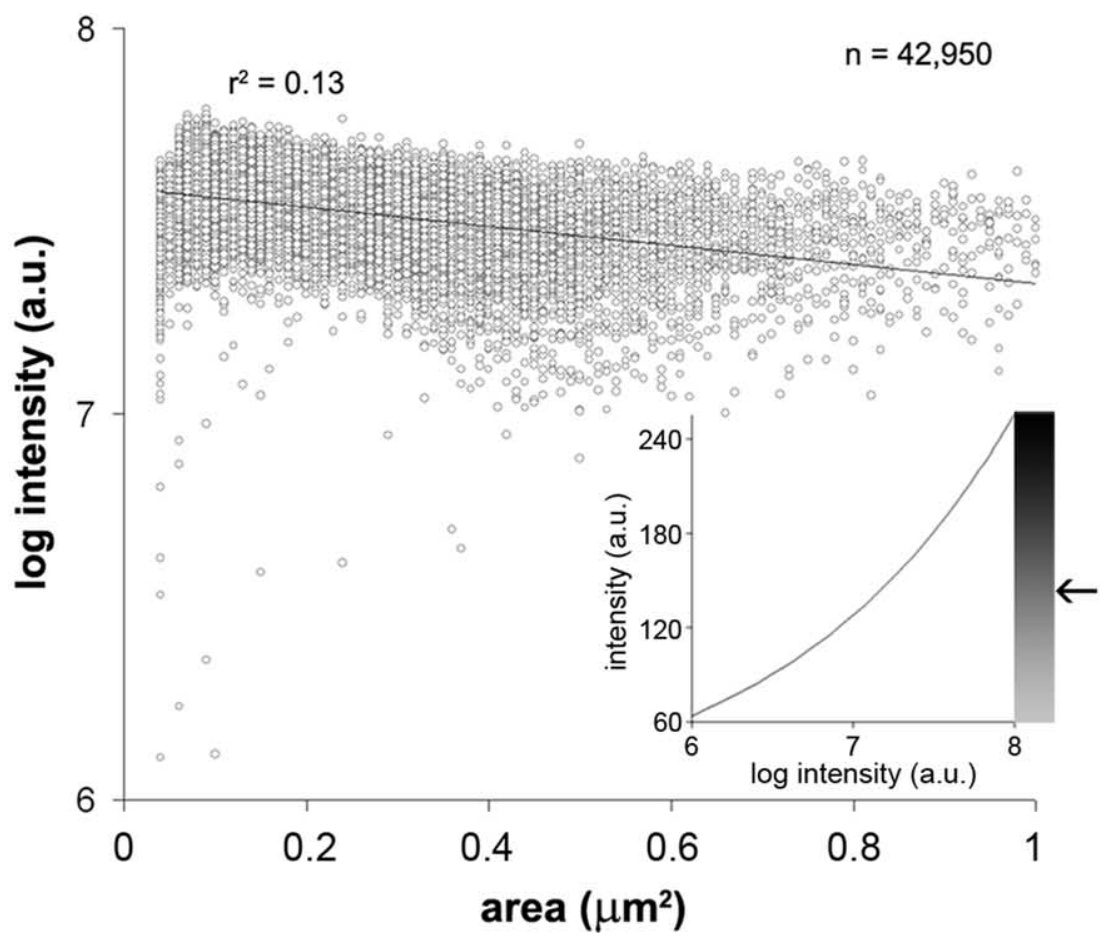

Figure 7. Fluorescence label intensity is not related to PSD-95-ir synapse area. $\boldsymbol{A}$, Photomicrographs at low power (left) and high power (right) show AlexaFluor 488 immunofluorescence labeling in hippocampal CA1 for PSD-95-ir puncta. sp, Stratum pyramidale; sr, stratum radiatum. Micrograph intensities were inverted for analysis (bottom right shows same area as the top right) and object identification was performed as described (see Materials and Methods); areas and mean intensities of identified PSD-95+ puncta were calculated by this process. $\boldsymbol{B}$, Plot of synapse area versus $\log _{2}$ mean intensity shows no correlation between the two measures $\left(r^{2}=0.13\right)$ on $\sim 43,000$ synapses sampled equally from control and experimental animals. The inset plot shows the log-linear relationship of label density; intensity scale corresponds to linear $(y)$ axis. The arrow indicates mean intensity of the synapse identified in $\boldsymbol{A}$. spines because the frequency curve for PSD areas was less skewed in the pCof + group, an observation made in previous work with hippocampal slices (Chen et al., 2007). Computer simulations suggested that both increased PSD area and a shifted area distribution could arise from a simple rounding of the synapses, with no necessary change in circumference. The similarity between the slice and behavioral results again suggests that the two are closely related. In all, the NMDA receptor initiated sequences responsible for phosphorylating cofilin are likely accompanied by a change in the shape and, hence, the area of the synapse.

Importantly, the differences between the frequency distributions for the pCofversus $\mathrm{pCof}+$ synapses were present in all three groups. This too has a parallel in the LTP experiments: pCof + spines were present, albeit in low numbers, before the induction of potentiation, and these spines had large, normally distributed synapses. Given that cofilin phosphorylation is a transient event, these results indicate that the potentiated state of the spine is not a unique consequence of LTP, but instead occurs spontaneously, although with very low probability, and reversibly, in hippocampal slices. By this argument, LTP involves both markedly increasing the probability that spines will enter the potentiated state and activating machinery that stabilizes the new spine configuration, with the latter step having a higher threshold. We propose that learning similarly transfers a small number of spines from one state, with relatively small synapses, to another with larger synapses. Whether it also engages actin polymerization and other mechanisms of LTP stabilization remains to be tested, although the presence of increased numbers of pCof + spines in the experimental group points in that direction.

It should be noted that the pCof + spines in the control groups ( $\mathrm{HC}$ and CPP) could have been induced by learning, rather than being the outcome of a spontaneous process. Although the transport of HC rats to the testing environment was an everyday occurrence, this event undoubtedly provided material for new memory. However, the $\mathrm{HC}$ and $\mathrm{CPP}$ groups had similar numbers of pCof + spines and it seems unlikely that these rats, exposed as they were to very different circumstances, experienced comparable amounts of learning. This argument suggests that the controls are dominated by a low rate at which spines spontaneously transition into a minutes-long, pCof + state. 
In addition to blocking the increase in pCofilin spines, CPP caused an evident disruption of long-term memory formation, as expected from what was observed previously in other behavioral studies (Ward et al., 1990; Mele et al., 1996) and its effects on LTP (Mele et al., 1995; Brun et al., 2001). More generally, there is considerable evidence that NMDA receptor antagonists block many, but not all forms of learning (Morris et al., 1986; Goosens and Maren, 2003). CPP produced its effects without significantly changing the amount of movement during day 1 of testing. Additional examination of the data, however, revealed that CPP caused behavioral changes that were unlikely to be consequences of blocking memory formation. The complex environment, as designed, includes subfields that appear to be ethologically significant locations for the rat [termed "places" in the study by Golani et al. (2005)], and provides attractive materials for investigation. Although testing rats in a laboratory environment necessarily restricts the animal's movements (Whishaw et al., 2006), this restriction can be exploited as a feature. Rats even in an undifferentiated (but spatially restricted) environment will establish a home base somewhere in the arena (Eilam and Golani, 1989), and will develop "preferred places" in which they linger (Tchernichovski et al., 1996). Designing likely candidates for such behaviors into the environment gives structure to the spontaneous exploratory behavior of individual animals, and allowed drug-induced disruption in this behavior to be revealed. Thus, without changing the amount of movement, CPP-treated rats used many more of their transitions between locations to return to the dark area than did controls, and as a result spent a smaller percentage of time exploring the open field and the objects within it. The possibility thus exists that the failure of the treated rats to exhibit day-to-day changes in the duration of their visits to the objects was in part or whole caused by a disruption of normal search patterns. Yet, the overall amount of day 1 activity, which was comparable with control levels, did not decrease on day 2 (in contrast to the controls). This suggests that the drug, in addition to altering movement patterns, produced a broad disruption of memory storage.

More detailed analyses of the relationship between learning and spine changes will require faster techniques for dealing with the very large dendritic territory that must be sampled to generate robust results. Our present methods do not allow for the use of numerous groups, each with a sizable number of rats, and so preclude experimental designs involving several time points. Ongoing work suggests that these problems can be reduced using automated microscopes and dedicated software; if so, then it will be possible in future studies to ask when during the $30 \mathrm{~min}$ behavioral sessions the LTP-related changes in spines first appear and if their magnitude is related to the complexity of the environment. It will also be important to develop actin-related markers that are more stable than phosphorylated cofilin. Slice studies have shown that phosphorylation persists for $<15$ min (Chen et al., 2007) and, thus, cannot be used to assess the status of spines in the hours after the learning session. Theta-burst induced poly- merization of spine actin lasts for hours in slices (Kramar et al., 2006), but the F-actin labeling method has proven difficult to use in vivo. Nonetheless, it is likely that modifications to this technique will permit investigations into the stability of the spine and synapse changes described in the present study.

Past studies have searched for the physical locus of memory by asking if nodal points in well defined circuits are affected by training, and if the location of such effects can be linked to specific features of the learning paradigm (e.g., the nature of the chosen conditioned stimulus) (Krupa et al., 1993). The present experiments constitute a first step toward the alternative approach of mapping broad areas of the telencephalon for the substrates of memory by marking individual synapses for events supporting the consolidation of LTP.

\section{References}

Blair HT, Sotres-Bayon F, Moita MA, Ledoux JE (2005) The lateral amygdala processes the value of conditioned and unconditioned aversive stimuli. Neuroscience 133:561-569.

Bliss TV, Collingridge GL (1993) A synaptic model of memory: long-term potentiation in the hippocampus. Nature 361:31-39.

Boda B, Nikonenko I, Alberi S, Muller D (2006) Central nervous system functions of PAK protein family: from spine morphogenesis to mental retardation. Mol Neurobiol 34:67-80.

Bramham CR, Messaoudi E (2005) BDNF function in adult synaptic plasticity: the synaptic consolidation hypothesis. Prog Neurobiol 76:99-125.

Brun VH, Ytterbo K, Morris RG, Moser MB, Moser EI (2001) Retrograde amnesia for spatial memory induced by NMDA receptor-mediated longterm potentiation. J Neurosci 21:356-362.

Carlisle HJ, Kennedy MB (2005) Spine architecture and synaptic plasticity. Trends Neurosci 28:182-187.

Chen LY, Rex CS, Casale MS, Gall CM, Lynch G (2007) Changes in synaptic morphology accompany actin signaling during LTP. J Neurosci 27:5363-5372.

Davis S, Bliss TV, Dutrieux G, Laroche S, Errington ML (1997) Induction and duration of long-term potentiation in the hippocampus of the freely moving mouse. J Neurosci Methods 75:75-80.

Doyere V, Laroche S (1992) Linear relationship between the maintenance of 
hippocampal long-term potentiation and retention of an associative memory. Hippocampus 2:39-48.

Eilam D, Golani I (1989) Home base behavior of rats (Rattus norvegicus) exploring a novel environment. Behav Brain Res 34:199-211.

Fukazawa Y, Saitoh Y, Ozawa F, Ohta Y, Mizuno K, Inokuchi K (2003) Hippocampal LTP is accompanied by enhanced F-actin content within the dendritic spine that is essential for late LTP maintenance in vivo. Neuron 38:447-460.

Gamlin PD, Reiner A, Erichsen JT, Karten HJ, Cohen DH (1984) The neural substrate for the pupillary light reflex in the pigeon (Columba livia). J Comp Neurol 226:523-543.

Ganeshina O, Berry RW, Petralia RS, Nicholson DA, Geinisman Y (2004) Differences in the expression of AMPA and NMDA receptors between axospinous perforated and nonperforated synapses are related to the configuration and size of postsynaptic densities. J Comp Neurol 468:86-95.

Golani I, Benjamini Y, Dvorkin A, Lipkind D, Kafkafi N (2005) Locomotor and exploratory behavior. In: Behavior of the laboratory rat: a handbook with tests (Whishaw IQ, Kolb B, eds), pp 171-182. New York: Oxford UP.

Goosens KA, Maren S (2003) Pretraining NMDA receptor blockade in the basolateral complex, but not the central nucleus, of the amygdala prevents savings of conditional fear. Behav Neurosci 117:738-750.

Granger R, Whitson J, Larson J, Lynch G (1994) Non-Hebbian properties of long-term potentiation enable high-capacity encoding of temporal sequences. Proc Natl Acad Sci USA 91:10104-10108.

Hayashi ML, Choi SY, Rao BS, Jung HY, Lee HK, Zhang D, Chattarji S, Kirkwood A, Tonegawa S (2004) Altered cortical synaptic morphology and impaired memory consolidation in forebrain- specific dominantnegative PAK transgenic mice. Neuron 42:773-787.

Hunt CA, Schenker LJ, Kennedy MB (1996) PSD-95 is associated with the postsynaptic density and not with the presynaptic membrane at forebrain synapses. J Neurosci 16:1380-1388.

Jain AK (1984) Fundamentals of digital image processing. Englewood Cliffs, NJ: Prentice Hall.

Juliano RL, Reddig P, Alahari S, Edin M, Howe A, Aplin A (2004) Integrin regulation of cell signalling and motility. Biochem Soc Trans 32:443-446.

Kramar EA, Lin B, Rex CS, Gall CM, Lynch G (2006) Integrin-driven actin polymerization consolidates long-term potentiation. Proc Natl Acad Sci USA 103:5579-5584.

Krupa DJ, Thompson JK, Thompson RF (1993) Localization of a memory trace in the mammalian brain. Science 260:989-991.

Lin B, Kramar EA, Bi X, Brucher FA, Gall CM, Lynch G (2005) Theta stimulation polymerizes actin in dendritic spines of hippocampus. J Neurosci 25:2062-2069.

Mele A, Castellano C, Cestari V, Oliverio A (1995) Interaction between the NMDA competitive antagonist CPP and the dopaminergic system in onetrial inhibitory avoidance in C57BL/6 mice. Neurobiol Learn Mem 63:143-148.

Mele A, Castellano C, Felici A, Cabib S, Caccia S, Oliverio A (1996) Dopamine- $N$-methyl-D-aspartate interactions in the modulation of locomotor activity and memory consolidation in mice. Eur J Pharmacol 308:1-12.

Morris RG, Anderson E, Lynch GS, Baudry M (1986) Selective impairment of learning and blockade of long-term potentiation by an $\mathrm{N}$-methyl-Daspartate receptor antagonist, AP5. Nature 319:774-776.

Neitz J, Jacobs G (1986) Reexamination of spectral mechanisms in the rat (Rattus norvegicus). J Comp Psychol 100:21-29.

Nusser Z, Lujan R, Laube G, Roberts JD, Molnar E, Somogyi P (1998) Cell type and pathway dependence of synaptic AMPA receptor number and variability in the hippocampus. Neuron 21:545-559.

Okamoto K, Nagai T, Miyawaki A, Hayashi Y (2004) Rapid and persistent modulation of actin dynamics regulates postsynaptic reorganization underlying bidirectional plasticity. Nat Neurosci 7:1104-1112.

Olds J, Disterhoft JF, Segal M, Kornblith CL, Hirsh R (1972) Learning centers of the rat brain mapped by measuring latencies of conditioned unit responses. J Neurophysiol 35:202-219.

Petersen JD, Chen X, Vinade L, Dosemeci A, Lisman JE, Reese TS (2003) Distribution of postsynaptic density (PSD)- 95 and $\mathrm{Ca}^{2+} /$ calmodulindependent protein kinase II at the PSD. J Neurosci 23:11270-11278.

Racca C, Stephenson FA, Streit P, Roberts JD, Somogyi P (2000) NMDA receptor content of synapses in stratum radiatum of the hippocampal CA1 area. J Neurosci 20:2512-2522.

Racz B, Weinberg RJ (2006) Spatial organization of cofilin in dendritic spines. Neuroscience 138:447-456.

Rex CS, Lin CY, Kramar EA, Chen LY, Gall CM, Lynch G (2007) Brainderived neurotrophic factor promotes long-term potentiation-related cytoskeletal changes in adult hippocampus. J Neurosci 27:3017-3029.

Reymann KG, Frey JU (2007) The late maintenance of hippocampal LTP: requirements, phases, "synaptic tagging," "late-associativity" and implications. Neuropharmacology 52:24-40.

Ribot T (1882) Diseases of memory. New York: Appleton-Century-Crofts.

Roman F, Staubli U, Lynch G (1987) Evidence for synaptic potentiation in a cortical network during learning. Brain Res 418:221-226.

Sarmiere PD, Bamburg JR (2004) Regulation of the neuronal actin cytoskeleton by ADF/cofilin. J Neurobiol 58:103-117.

Schacter D (1982) Stranger behind the engram: theories of memory and the psychology of science. Hillsdale, NJ: Erlbaum. Semon R (1921) The mneme. London: Allen and Unwin.

Semon R (1921) The mneme. London: Allen and Unwin.

Takumi Y, Ramirez-Leon V, Laake P, Rinvik E, Ottersen OP (1999) Different modes of expression of AMPA and NMDA receptors in hippocampal synapses. Nat Neurosci 2:618-624.

Tchernichovski O, Benjamini Y, Golani I (1996) Constraints and the emergence of "free" exploratory behavior in rat ontogeny. Behaviour 133:519-539.

Ward L, Mason SE, Abraham WC (1990) Effects of the NMDA antagonists CPP and MK-801 on radial arm maze performance in rats. Pharmacol Biochem Behav 35:785-790.

Whishaw IQ, Gharbawie OA, Clark BJ, Lehmann H (2006) The exploratory behavior of rats in an open environment optimizes security. Behav Brain Res 171:230-239.

Whitlock JR, Heynen AJ, Shuler MG, Bear MF (2006) Learning induces long-term potentiation in the hippocampus. Science 313:1093-1097.

Zhou Q, Homma KJ, Poo MM (2004) Shrinkage of dendritic spines associated with long-term depression of hippocampal synapses. Neuron 44: 749-757. 